\title{
A systematic review of antiproton radiotherapy
}

\author{
Martin-Immanuel Bittner ${ }^{1}{ }^{*}$, Anca-Ligia Grosu ${ }^{1}$, Nicole Wiedenmann ${ }^{1}$ and Jan J. Wilkens ${ }^{2}$ \\ ${ }^{1}$ Department of Radiation Oncology, University Medical Center Freiburg, Freiburg, Germany \\ ${ }^{2}$ Department of Radiation Oncology, Klinikum Rechts der Isar, Technische Universität München, München, Germany
}

\section{Edited by:}

Jaakko O. Nieminen, Aalto

University, Finland

Reviewed by:

Otto Muzik, Wayne State University, USA

Edite Figueiras, Tampere University of Technology, Finland

Jonathan O. De Xivry, Université

Catholique de Louvain, Belgium

\section{*Correspondence:}

Martin-Immanuel Bittner.

Department of Radiation Oncology,

University Medical Center Freiburg,

Robert-Koch-Str. 3, D-79106

Freiburg, Germany

e-mail:martin-immanuel.bittner@

uniklinik-freiburg.de
Antiprotons have been proposed as possible particles for radiotherapy; over the past years, the renewed interest in the potential biomedical relevance led to an increased research activity. It is the aim of this review to deliver a comprehensive overview regarding the evidence accumulated so far, analysing the background and depicting the current status of antiprotons in radiotherapy. A literature search has been conducted, including major scientific and commercial databases. All articles and a number of relevant conference abstracts published in the respective field have been included in this systematic review. The physical basis of antiproton radiotherapy is complex; however, the characterization of the energy deposition profile supports its potential use in radiotherapy. Also the dosimetry improved considerably over the past few years. Regarding the biological properties, data on the effects on cells are presented; however, definite conclusions regarding the relative biological effectiveness cannot be made at the moment and radiobiological evidence of enhanced effectiveness remains scarce. There is new evidence supporting the potential imaging properties, for example for online dose verification. Clinical settings which might profit from the use of antiprotons have been further tracked. Judging from the evidence available so far, clinical constellations requiring optimal sparing in the entrance region of the beam and re-irradiations might profit most from antiproton radiotherapy. While several open questions remain to be answered, first steps toward a thorough characterization of this interesting modality have been made.

Keywords: antiprotons, particle therapy, hadron therapy, radiobiology, online range verification

\section{INTRODUCTION}

Radiotherapy aims at controlling tumors by delivering sufficiently high doses to achieve a high probability of killing all tumor cells while at the same time not harming adjacent tissues. The modalities mostly used for irradiation are photon and electron beams. Over the past decades, proton therapy—as a form of particle therapy with particles heavier than electrons-has evolved as another beam modality with increasing availability [1]. In addition, several other particles have been or are currently being assessed, such as pions, neutrons, carbon ions or other heavy particles [2]. Another modality, which has been proposed as possibly superior to other existing and developing therapies, is antiproton radiotherapy [3, 4]. However, the potential advantages and disadvantages of antiproton radiotherapy have been subject to ongoing debate.

There are currently only very few facilities worldwide where antiprotons can be produced. The most advanced currently active facility is located at CERN (Geneva, Switzerland). The antiproton production at Fermilab (Batavia, USA) has stopped after closure of the Tevatron in 2011. In general, the production of antiprotons is a very costly and time-consuming process requiring immense investments in special facilities and their maintenance [5]. In addition, the current antiproton fluence rates achievable are relatively low and require a long time to build up relevant (>1 Gy) doses [6-9]; the experimental conditions are still not suitable for in vivo experiments [10]. The current flux at the
CERN facility is $3 \times 10^{7}$ antiprotons every $90 \mathrm{~s}$; however, $4 \times 10^{11}$ antiprotons are needed to deliver a dose of $20 \mathrm{~Gy}$ to $100 \mathrm{~cm}^{3}$ [11]. Considering these technological (and economic) issues, it can be stated that antiproton radiotherapy in any case will only be available for very special indications and certainly not in the near future. However, new sites to be opened like the FAIR project at the GSI (Darmstadt, Germany), might offer the potential to study clinical implications in more detail over the next years.

Antiproton radiotherapy has first been proposed in 1982, and in 2008 the first and only detailed review [12] on antiproton radiotherapy was published so far. However, within the past 6 years, numerous new articles and abstracts have been published. Therefore, this review aims at giving an independent, comprehensive overview regarding the current state of antiproton radiotherapy research. This is deemed necessary when taking into account the renewed interest in this modality. In the course of this article, the physical and biological basis as well as the latest findings will be discussed, while also summarizing and re-examining possible clinical implications: primarily in therapy, but also in imaging, taking advantage of the particle spectrum coming into existence during the annihilation process.

\section{METHODS OF LITERATURE REVIEW}

A literature review was performed between October 2012 and April 2013 using scientific and medical as well as publishers' databases: PubMed/Medline, BIOSIS, DAHTA, EMBASE, EBSCO 
Host, OvidSP, Elsevier ScienceDirect, SciSearch, Thomson Reuters (formerly ISI) Web of Knowledge, Scopus, gms, Thieme, and Karger.

In addition, an open internet search using the most common web search engines (google.com and scholar.google.com, yahoo.com, bing.com) was done. Keywords included antiproton, antiproton therapy, antiproton radiotherapy, antiproton radiation therapy and antiproton imaging.

During the course of the review process, the reference lists of all articles included were also hand searched to detect any further articles or abstracts potentially relevant. However, master theses or doctoral theses were not included.

In the end, this systematic review was based on one comprehensive review article, one editorial, one status report and 37 original articles and/or conference abstracts specifically addressing the biomedical perspectives of antiproton beams. To our best knowledge this is the first review since 2008 covering all existing and accessible scientific works in this field and summarizing and reviewing them. In addition, several background articles (e.g., regarding hadron therapy and range verification as well as related topics), were also included in writing this review. We therefore deem our work the first systematic review of the evidence on antiproton radiotherapy collected so far, to our best knowledge based on all articles as well as most abstracts available.

\section{CURRENT STATUS OF RESEARCH PHYSICAL ASPECTS}

Antiprotons are the antimatter counterpart of protons. This means that these particles share the same characteristics (for example the same mass), except for an opposite charge. Antimatter particles annihilate with their matter counterparts by liberating the energy corresponding to their respective masses. Thus, energy deposition consists of two components: stopping energy corresponding to the complete loss of kinetic energy until coming to rest (according to Bethe-Bloch), which is shared by protons, antiprotons and other particles, and annihilation energy, which is an exclusive feature of antimatter particles and will be discussed below. The stopping energies, however, are given for a specific kinetic energy and range and thus have to be compared using the same values for different particles.

As for other particle beams, there are certain characteristics which differ from the clinically more common photon beams. These include the beam profile, which forms a Bragg peak, and the secondary particles or fragments which play a role in the biological effects and will be elaborated on.

A biomedical application of antimatter which is relatively widespread is positron emission tomography, which involves detecting the $511 \mathrm{keV}$ photons characteristically emitted when a positron and its counterpart, the electron, annihilate.

The annihilation energy of an antiproton and a proton on the other hand is much higher, amounting to $1.88 \mathrm{GeV}$. This energy is distributed among a variety of annihilation particles which either emerge from the annihilation itself or from reactions with nearby atoms. The most important groups of particles are charged and uncharged pions (together around $80 \%$ of the energy), neutrons, photons, heavy charged particles (ions of various nuclear charge and mass) and a small amount of other particles such as electrons, kaons and neutrinos $[4,13]$. Pions and kaons belong to the family of mesons, which are hadrons (like protons and neutrons), but do only consist of two instead of three quarks. They have a mass, but are short-lived. In contrast, neutrinos are leptons, practically massless and hardly interacting with other matter. See also Table 1 for an overview of particles important in this context. This variety of particles can further be discriminated by half-lives, interaction patterns etc. However, it is important to note that the major biological effects in the target are due to the heavy particles with their high linear energy transfer (LET), although the exact composition of these particles is unknown $[12,14]$. Most other particles leave the body without further interactions. Some particles, however, do interact in the body outside the target. In this regard, special attention has to be paid to neutrons, because of their relatively high biological effectiveness $[6,15]$. See also Figure 1 for an illustration of the energies and respective particle species deposited in certain volumes within and outside of the body.

When considering the diversity of the annihilation products, it is important to know how much energy will be deposited locally, i.e., in the target volume. The estimations for this proportion varied between less than $30 \mathrm{MeV}$ [16] and up to $150 \mathrm{MeV}$ [4]. The latest experimental data and simulations point toward $30 \mathrm{MeV}$ being deposited in the immediate vicinity of the annihilation [13]. This amount of energy increases with increasing field size, due to more secondary particles coming to rest within this increased radius; on the other hand, increasing field sizes also increase the amount of secondary particles coming to rest in other parts of the body, hereby increasing the dose halo [13].

Dosimetry in the mixed particle field of antiproton annihilation is generally difficult [17]. The latest studies report of advanced (relative) dose measurements using ionization chambers [18], because earlier measurements with thermoluminescent devices and radiochromic films were not promising due to the lack of models linking detector responses to actual dose values [12]. Using alanine detectors for (absolute) dosimetry might be another option, both for the mixed spectrum peak region and the plateau [19]. For beam monitoring (also in a possible clinical setting), monolithic active pixel sensors made of crystalline silicon have been proposed and shown first promising results [20, 21]. A detailed description of currently employed sensor systems (and the set-up of cell irradiation experiments) was given by Sellner et al. [21]. It has to be highlighted that adequate dosimetry is also very important to be able to define relative biological effectiveness (RBE) values in preclinical studies.

In addition, in-flight processes have to be distinguished from at-rest processes. In flight, antiprotons are very comparable to protons [18]. They do interact slightly more, in particular by in-flight annihilation events. Originally thought to be a rather rare event $[4,16]$ in fact it seems to play a non-negligible role, especially at higher beam energies [13]. In-flight annihilation is becoming more important when the normal tissue the beam has to transverse before entering the target volume increases; for a planning target volume (PTV) in a depth of $10 \mathrm{~cm}$, a loss of $25 \%$ of the antiprotons has been calculated [22]—which is, however, still less than the loss of primary carbon ions due to fragmentation in a corresponding setting with a carbon ion beam. 
Table 1 | Overview of important particles involved in antiproton radiotherapy.

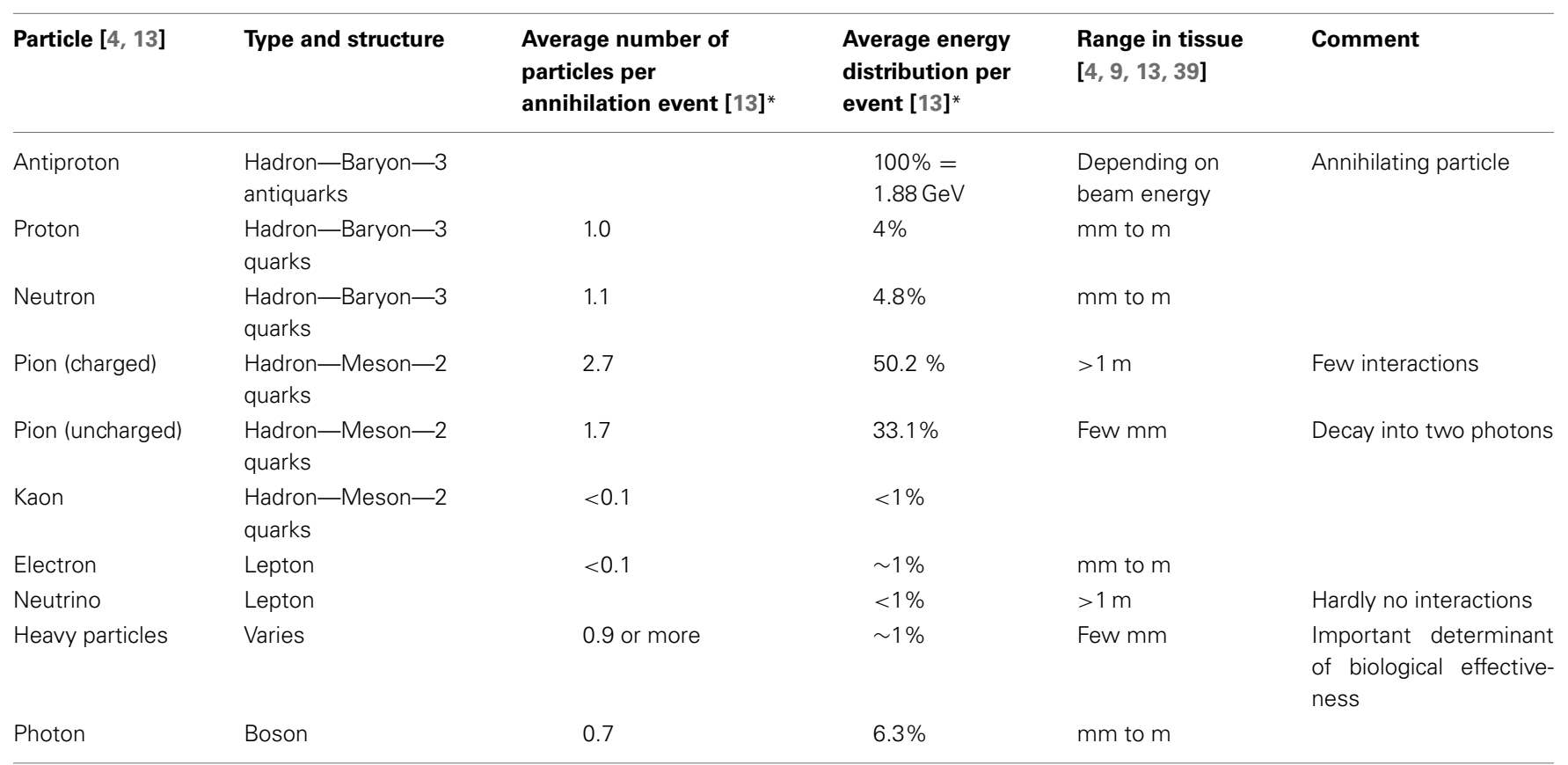

*for a $120 \mathrm{MeV}$ beam, annihilation at rest, in water.

When slowing down and coming to rest, the antiprotons eventually annihilate on a matter particle- the higher the mass, the more probable this event is [12]. The depth of the majority of the annihilation events, i.e., the length of the distance in the body, is subject to the energy of the antiproton beam and can be delineated very sharply, forming a Bragg peak. The depth-dose curve in water showed the antiproton Bragg peak being twofold higher compared to a proton beam of the same size and energy while at the same time showing only a slight elevation along the entry region [18]. A spread-out Bragg peak (SOBP) can be formed by variation of the beam energy, thereby covering a potential target volume.

When spreading out the beam, a number of parameters change. As mentioned above, the number of secondary particles increases, which is also the reason for the dose halo and the lateral penumbra to increase. In addition, it is known from other particles that when broadening the peak or modulation width, the difference in the RBE between peak and plateau (i.e., entrance region) will be reduced $[13,23,24]$. However, for antiprotons the interactions between RBE, LET and beam physics and the influence of changes on the dose distribution and the biological effects are only poorly characterized so far.

\section{BIOLOGICAL ASPECTS}

Considerations regarding potential radiobiological advantages of antiprotons were primarily based on the increased energy deposition (see "Physical aspects") and on the heavy, high LET particles causing damage in the immediate vicinity of the annihilation point; a low oxygen enhancement ratio has also been attributed [25], but stands without experimental evidence.

A number of experiments with V79 Chinese hamster cells $[7,11,26,27]$ have been administered at the CERN antiproton facility in Geneva, Switzerland. The first experiments suffered from the lacking possibility to adequately consider the physical depth-dose distribution of the antiproton beam. The later experiments, however, revealed important information about the biological effects of antiproton beams. Using clonogenic survival and Comet assays, it has been shown that the dose fall-off behind a pristine Bragg peak is very sharp [11]: Within few millimetres, the Comet assay returns to baseline values and the clonogenic survival returns to $90 \%$. However, it has to be annotated that peripheral damage due to more long-range particles such as neutrons has not been analyzed in this experimental set-up with sampling being restricted to approximately $30 \mathrm{~mm}$ distance from the irradiation peak. Due to the challenges in dosimetry, RBE values could only be estimated partly using values calculated through simulations and after implementing a quantity, defined as the ratio of fluences rather than doses, thereby dispensing of the need to assess the absolute dose [7]. When making several assumptions, this allows an estimate of the RBE, because fluence and dose are directly proportional. Following this approach, the RBE estimate for antiprotons in a $2.5 \mathrm{~mm}$ SOBP for a $46.7 \mathrm{MeV}$ pulsed beam was reported to be 2.25 [7], with a peak-to-plateau dose ratio of 4 , both values roughly twice as high as for protons.

The afore-mentioned assumptions were first that the antiproton RBE in the plateau (i.e., the in-flight RBE) is equal to that of protons $[28,29]$ and therefore $1[30]$ and second that the Monte Carlo simulation of the antiproton dose ratio is accurate enough.

However, the LET influences the RBE and therefore also has to be addressed. This explains that once the LET was known, RBE values from other experiments had to be re-evaluated. For the Bragg peak, the LET has recently been found to be around $19 \mathrm{keV} / \mu \mathrm{m} \mathrm{[28];} \mathrm{it} \mathrm{has} \mathrm{been} \mathrm{indicated} \mathrm{that} \mathrm{the} \mathrm{RBE} \mathrm{in} \mathrm{the} \mathrm{plateau}$ can be assumed to be around $1.2-1.3$, instead of the 1 originally 


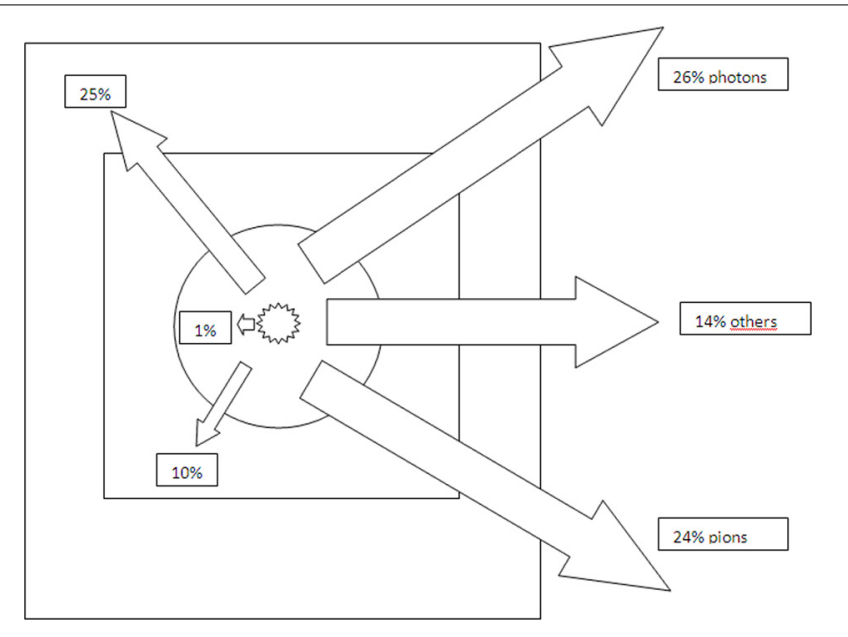

FIGURE 1 | Energy deposition (particle rest mass and kinetic energy of the antiproton) in relation to particle type and location relative to the annihilation point (in percent) [13]. The circle denotes the region within $5 \mathrm{~mm}$ of the annihilation point, the inner rectangle the planning target volume $\left(10 \times 10 \times 10 \mathrm{~cm}^{3}\right)$ and the outer rectangle the water phantom $\left(50 \times 50 \times 150 \mathrm{~cm}^{3}\right)$. With increasing distance from the point of annihilation, the contribution towards energy deposition of protons, pions and electrons increases, whilst the proportion of heavy particles decreases.

expected. This means the RBE in the peak region, which is expressed in relation to the RBE in the plateau region also had to be re-estimated: instead of 2.25 expected so far [7], it might rather be 2.7-3.0 (again, for very small SOBPs) [28]. The accuracy of the estimates for the RBE values may be improved when the plans to re-evaluate all experiments done so far is put into effect [31].

The latest biological experiments exposed human fibroblasts to antiproton radiation and also analyzed possible bystander effects [8]: The gamma-H2AX assay, a sensitive method to show DNA damage appearing as foci, revealed that there was considerable DNA damage in the peak region which appeared to be qualitatively different from the damage in the plateau. The second technique used was a plasmid DNA solution. Different forms of DNA damage result in different forms of fragments which can be separated electrophoretically. This revealed a majority of double-strand and complex breaks caused by antiprotons. Other samples of cells were not directly irradiated with the antiproton beam but either exposed to secondary particles or exposed to secondary particles while sharing their medium with irradiated cells. However, no significant effects were found for either samples. Inconsistent results may in part be due to small sample sizes and suboptimal experimental conditions, including long transport times of biological material to and from the laboratory facilities [8].

Secondary particles generated in the annihilation event can increase the dose in other parts of the body. Among these particles, neutrons attracted most attention so far, regarding their high biological effectiveness. Their measurement is difficult, but thermoluminescent detectors seem to provide reliable results for thermal neutron fluence [9]. Bubble detectors have been used for the measurement of fast neutron fluence, but are of limited applicability in mixed particle fields [15]. According to the current understanding, thermal neutrons contribute only insignificantly to dose outside the target volume [9]. High energy neutrons, however, do have a significant dose-increasing effect in distant parts of the body, where the respective organ dose depends on irradiation field geometry and distance to the target volume $[32,33]$. The equivalent dose has been estimated to be around 60 times higher than for a comparable proton therapy treatment plan for a cylindrical intracranial target volume of $1 \mathrm{~cm}$ diameter and $1 \mathrm{~cm}$ length [33]. However, the delivery technique used is also supposed to have a substantial influence on this relative increase [34]. In addition, the dose distributed by neutrons is increasing with the target volume, which has led to the conclusion that antiprotons in general might only be suitable for irradiating relatively small structures [9]. However, the calculations presented have to be interpreted cautiously, taking into account that the adequacy of the currently used weighting factors for secondary neutrons evolving from proton treatment has also been questioned [35]. Therefore, at the moment no definite conclusions on the effects of the secondary neutrons can be drawn. Further neutron calculations using benchmarked Monte Carlo codes and stating results according to established ICRP protocols are to be recommended. The physical dose contributions to other parts of the body due to pions and photons is similar to the neutron dose, but because of the low LET, their equivalent dose is supposed to be significantly smaller [33]. Monte Carlo simulations have recently been proposed to estimate the peripheral dose to be expected [36].

\section{IMAGING ASPECTS}

It has also been suggested that antiprotons could not only be used for therapy, but also for imaging purposes [3, 37]. Particles evolving through the annihilation process can be detected when escaping the body and used to define their point of origin by extrapolating the trajectories found when hitting the detectors. The most intriguing advantage would be the control of the annihilation position and thus the point of energy delivery. It has also been proposed to confirm the accuracy of patient-positioning with antiprotons, directly followed by the application of any charged particle beam (as long as it is corrected for mass and charge) [37]. However, this proposed calibration still represents a hypothetical concept. In contrast, the possibility of real-time imaging for online dose verification during delivery of antiprotons as therapeutic modality has further been studied more recently [38-40]. Computer simulations using FLUKA showed that photons and charged pions could both be used for imaging purposes. These two annihilation products differ in their characteristics: Photons, in most cases results of a neutral pion decay happening in the timescale of less than a femtosecond, scatter to less than $20 \%$ when their energy is higher than $10 \mathrm{MeV}$. In addition, the energy spectrum offers a prominent peak at $511 \mathrm{keV}$, which is due to positron emission by excited nuclei and consecutive annihilation. Therefore, both photon populations might be feasible for realtime imaging, noting that the half-life of the excited nuclei is prolonged, which may result in imaging delayed by a certain factor of time (around tenths of seconds) [39]. Charged pions, on the other hand, scatter to a higher degree but are not subject to delay. Due to scattering, detector positioning and particles stopping in the patient, the overall efficiency of charged pion-detection in a 
hypothetical sophisticated detector set-up is around 1\%, however this still implies that a fraction of the clinically applied dose provides sufficient numbers of pions for reliable statistics [38], as has been hypothesized before [37]. When only the proportion with kinetic energies higher than $250 \mathrm{MeV}$ is selected, the accuracy of the location of the annihilation point by extrapolating the tracks can be improved to up to $+/-1 \mathrm{~mm}$. This means that the exact location of the field and the SOBP may be determined [38, 39]. The feasibility of another, more realistic detector set-up has also been studied; especially when assuming a continuous rather than a pulsed antiproton beam currently available, the accuracy has also been in the range of few millimetres, while at the same time confirming that only a fraction of the therapeutically administered antiprotons may be needed for imaging [40]. Taken together, these findings support the notion of an online dose or range verification during the treatment. However, the literature data regarding the exact corresponding dose are inconclusive, rendering comparisons with other concepts difficult. These existing concepts for dose and range verification during proton or carbon ion therapy are based on measurements of generated positron emitting nuclei $[41,42]$ or prompt gamma emission following nuclear interactions of the primary particles [43].

There is one other possible imaging application of antiprotons: The term ASTER - antiprotonic stereography_has been coined to describe the theory and hypothetical technique according to which antiprotons can also be used to deliver three-dimensional insights into body structures [37]. There are two different effects at the basis of this possible application which have led to the differentiation between ASTER-1 and ASTER-2 [44]. First, the amount of charged pions coming into existence corresponds with the number of annihilaton events, thus the density of the respective material. Reconstructing pion tracks can therefore help to make assumptions about the density structures of the material under investigation (ASTER-1). Second, the energy spectrum of photons emitted during the annihilation can show characteristic properties corresponding with the specific elements capturing the antiproton. This means, the chemical properties can be investigated (ASTER-2). It has to be noted that resolution and contrast are highly dependent on the number of antiprotons used and can thus be adapted to different tasks. Other advantages include a very low radiation dose received by the structure and neighboring tissues-estimated to be lower than a conventional CT doseand the possibility to specifically look at certain organs, thereby sparing other structures. However, this application has not been further studied yet.

\section{CLINICAL ASPECTS}

First proposals for possible clinical indications were rather wide; the antiproton was seen as a particle superior to all others [4]. With increasing knowledge and understanding, the settings with possible advantages for antiprotons narrowed. In 2008, the possible role for antiprotons in radiotherapy has been seen as potentially useful for three groups of patients [12]: First, re-irradiation of patients with local failure after radiotherapy (taking advantage of a potential sparing of surrounding tissues). Second, small tumors with organs-at-risk directly neighboring (potentially improved sparing again). Third, therapy-resistant subvolumes in a tumor (using the unique radiobiological properties of antiprotons with their high RBE). Two years later, a comparison of antiproton, proton and carbon ion treatment plans in a water phantom with a PTV of $4 \times 4 \times 4 \mathrm{~cm}^{3}$ surrounded by $1 \times 1 \times$ $1 \mathrm{~cm}^{3}$ volumes of interest was published [45]. This simulation revealed that antiprotons have the highest peak-to-plateau ratio, but do deposit dose beyond the PTV; carbon ions in comparison have a longer tail, whereas protons deliver no dose at all beyond the PTV. Outside the PTV, the dose from antiprotons spreads isotropically whereas carbon ions contain a directional component. Antiprotons deliver the lowest dose to the entrance region and the highest to the PTV, thereby increasing the dose lateral and beyond the PTV. The mean dose to the whole phantom (excluding the PTV) for the antiproton beam has been calculated to be $3.6 \%$ of the PTV dose, thus being twice as high as for the other particles. This study used laterally modulated fields to improve the target coverage. In contrast, Paganetti et al. [13] had used flat and also larger fields $\left(10 \times 10 \mathrm{~cm}^{2}\right)$, which deliver worse results in terms of target coverage and normal tissue sparing. Since the dose volume histograms for antiprotons and carbon ions only differed slightly in the treatment plan comparison by Bassler et al. [45], it has been concluded that only big differences in the RBE could compensate for the larger lateral penumbra and the background dose faced when using antiprotons.

An important question arising in the studies is which field or target size can be seen as clinically realistic. Most studies performed so far concentrated on narrow beams. However, the resulting, very small field sizes tend to underestimate the normal tissue dose due to secondary particles - a problem, which is much more pronounced for antiprotons than for protons [13]. Even when stating that clinically relevant target volumes in antiproton radiotherapy will rarely reach the dimensions of one liter, it has to be kept in mind that at least some increase in background dose has to be expected. A general limitation of most of the studies performed so far and reported here is the simplistic setting with cubic water phantoms; this clearly has to be kept in mind when interpreting the results.

The summarized results can lead to the conclusion that antiprotons probably offer advantages only in those cases where the normal tissue in the entrance region is of special importance. In this context, it has to be highlighted that a typical peak-to-plateau ratio for a proton beam is around 5-6, for a carbon ion beam similar, maybe higher (depending on beam line physics, RBE and other factors) and for an antiproton beam of the same energy around 9-10 [18, 46, 47]. Please see also Figure 2 for a schematic illustration of the depth dose profiles of photon, proton and antiproton beams. This ratio is very favorable for antiprotons, but it worsens with increasing beam energy $[13,48]$. Many other characteristics also depend on the beam energy used: Besides the range and the peak-to-plateau ratio, this is also true for the in-flight annihilation probability, the secondary particle spectrum [46] and radiobiological effects [45]. Recently, energy and intensity modulated treatment plans based on Monte Carlo simulations have been shown to be feasible for antiprotons [49].

A possible modification of antiproton radiotherapy has been proposed by Shmatov [50]: When heavy elements such as 


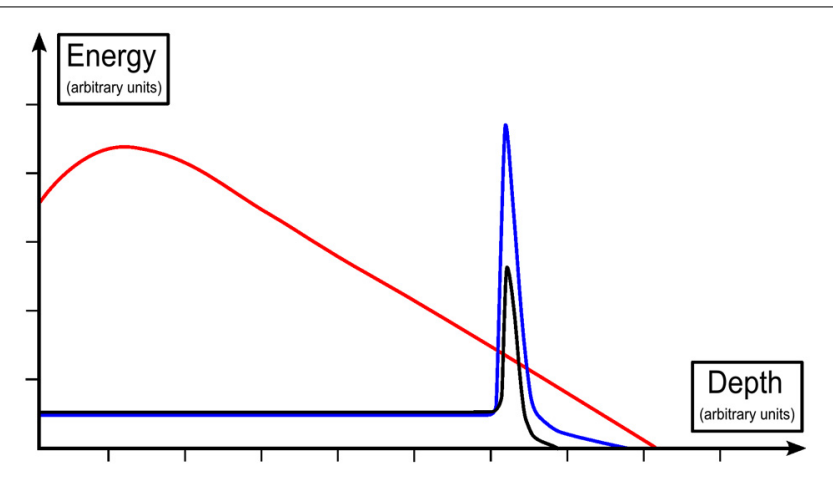

FIGURE 2 | Schematic display of depth dose profiles of a photon (red), proton (black) and antiproton beam (blue), with deposited energy expressed relative to the plateau region $[19,45]$.

Uranium-238 or Thorium-232 are brought into a tumor, the biologically effective dose administered with a given antiproton radiotherapy may be increased by $10 \%$ or more. This is explained with an increased amount of short-range particles due to the annihilation with the heavy elements and maybe also a reduced annihilation probability with other nuclei. However, these are only hypotheses lacking experimental data and not taking into account clinical complexities such as the effective introduction of the named substances into the tumor and toxicities potentially associated with it. In addition, irradiating heavier elements has also been suggested to lead to a smearing of the irradiated spot due to an increased neutron yield [51].

Considering the scarcity of (actual and proposed) antiproton producing centers worldwide, it can be hypothesized that clinical usage of antiprotons will in any case be very restricted. This is also due to the enormous costs imposed by antiproton production. However, the application of antiprotons may be facilitated-both from a practical and economic perspective-if the idea of portable antiproton traps finds its way into practical use $[52,53]$. This technique, based on the trapping of antiprotons through electrode potentials (vertical direction) and magnetic fields (radial direction) in an electron-cooled low energy state, might have the potential to render at least some of the biomedical applications possible in various sites. However, at the moment these are also mainly theoretical considerations and it is unknown, if and when this technology could be used in a clinical setting.

\section{CONCLUSIONS AND OPEN RESEARCH QUESTIONS}

When taking into account the simulation results generated over the past years as well as the latest experimental findings, the following conclusions can be drawn: The peak-to-plateau ratio as one of the major advantages of the antiproton beam properties is still a highly valuable feature. However, the lateral penumbra and increased dose halo compared to other particle beams reduce the applicability of antiprotons to very few possible indications. There may be clinical settings-especially when the normal tissue in the entrance region of the beam is highly radiosensitive-where antiproton radiotherapy offers special advantages. Possible clinical constellations include the treatment of recurrences, when the normal tissue has already received the maximum tolerable dose, and the dose in the entry channel has to be minimized. These settings have to be further analyzed by generating and comparing treatment plans and the respective dose distributions, based on assessments of the physical qualities of antiprotons in comparison with the different beam types available so far. In addition, the possibilities for real-time dose range verification offer a new feature in contrast to other particle beams which might attract more attention in the future. Other open questions exist regarding the role of secondary neutrons, which should be investigated following the appropriate ICRP protocols. Also, the biological properties of the antiproton beam have to be further characterized considering different cell lines and target sizes, as well as the effects of the medium- and long-range secondary particles.

In particular, the RBE-which depends on correct dosimetry-has to receive further attention. This has to be seen as a prerequisite for meaningful treatment planning studies, because as long as the biological properties are not validated, antiproton treatment planning cannot be performed. But only then will it be possible to think about in vivo experiments, which in turn require new facilities providing adequate fluence rates. Finally, the most important quantity is the ratio of the biologically effective dose in the target volume and the biologically effective dose to the normal tissue. This in turn has to be evaluated carefully in terms of cell killing (e.g., in the tumor) and of induction of secondary tumors (in the normal tissue).

Hence, there are still many open questions which need to be addressed by future research.

Given these uncertainties, no timeframe or schedule for an implementation into clinical practice can be predicted. However, research over the past decade did show progress in variousphysical, biological, imaging and clinical-aspects. Therefore, it can be anticipated that there will be an equaling number of new results over the next decade. Despite the still unrealistic technological implementation, the increasing number of pertinent publications and also patents [54] shows an ongoing academic interest in antiproton radiotherapy, reinforcing this notion. It is hoped that further experimental work will provide robust data for further elucidating the aforementioned questions and will back up the data generated in the various simulations so far. Only then will it be possible to draw definite conclusions on the potential role of antiprotons in clinical practice, and-in consequence-to evaluate the potential for an implementation of clinical usage.

\section{AUTHORS CONTRIBUTIONS}

Martin-Immanuel Bittner developed the idea for this review, carried out the literature review and drafted the manuscript. AncaLigia Grosu and Jan J. Wilkens participated in the design of the review and helped to draft the manuscript. Nicole Wiedenmann contributed through discussion of key findings. All authors read and approved the final manuscript.

\section{ACKNOWLEDGMENTS}

The article processing charge was funded by the German Research Foundation (DFG) and the Albert Ludwigs University Freiburg in the funding programme Open Access Publishing. 


\section{REFERENCES}

1. Nyström H. The role of protons in modern and biologically-guided radiotherapy. Acta Oncol. (2010) 49:1124-31. doi: 10.3109/0284186X.2010.498436

2. Slater JM. Considerations in identifying optimal particles for radiation medicine. Technol Cancer Res Treat. (2006) 5:73-9.

3. Gray L, Kalogeropoulos TE. Possible bio-medical applications of anti-protons I. In vivo direct density measures: radiography. IEEE Trans Nucl Sci. (1982) NS-29:1051-7. doi: 10.1109/TNS.1982.4336313

4. Gray L, Kalogeropoulos TE. Possible bio-medical applications of antiproton beams: focused radiation transfer. Radiat Res. (1984) 97:246-52. doi: $10.2307 / 3576276$

5. Kirchner T, Knudsen H. Current status of antiproton impact ionization of atoms and molecules: theoretical and experimental perspectives. J Phys B (2011) 44:122001. doi: 10.1088/0953-4075/44/12/122001

6. Hall EJ. Antiprotons for radiotherapy? Radiother Oncol. (2006) 81:231-2. doi: 10.1016/j.radonc.2006.09.003

7. Holzscheiter MH, Bassler N, Agazaryan N, Beyer G, Blackmore E, DeMarco JJ, et al. The biological effectiveness of antiproton irradiation. Radiother Oncol. (2006) 81:233-42. doi: 10.1016/j.radonc.2006.09.012

8. Kavanagh JN, Currell FJ, Timson DJ, Holzscheiter MH, Bassler N, Herrmann $\mathrm{R}$, et al. Experimental setup and first measurement of DNA damage induced along and around an antiproton beam. Eur Phys J D (2010) 60:209-14. doi: 10.1140/epjd/e2010-00194-4

9. Bassler N, Holzscheiter MH, Petersen JB. Neutron fluence in antiproton radiotherapy, measurements and simulations. Acta Oncol. (2010) 49:1149-59. doi: 10.3109/0284186X.2010.501812

10. Bassler N. Antiproton radiotherapy: 9th Biennial ESTRO meeting on physics and radiation technology for clinical radiotherapy. Barcelona. Radiother Oncol. (2007) 84:S22-3.

11. Kovacevic S, Bassler N, Hartley O, Knudsen H, Vranjes S, Garaj-Vrhovac V, et al. V-79 Chinese hamster cells irradiated with antiprotons, a study of peripheral damage due to medium and long range components of the annihilation radiation. Int J Radiat Biol. (2009) 85:1148-56. doi: 10.3109/09553000903242081

12. Bassler N, Alsner J, Beyer G, DeMarco JJ, Doser M, Hajdukovic D, et al. Antiproton radiotherapy. Radiother Oncol. (2008) 86:14-9. doi: 10.1016/j.radonc.2007.11.028

13. Paganetti H, Goitein M, Parodi K. Spread-out antiproton beams deliver poor physical dose distributions for radiation therapy. Radiother Oncol. (2010) 95:79-86. doi: 10.1016/i.radonc.2009.03.020

14. Knudsen HV, Holzscheiter MH, Bassler N, Alsner J, Beyer G, DeMarco JJ, et al. CERN ACE collaboration: antiproton therapy. 14th international workshop on low-energy positron and positronium physics. Reading. Nucl Instrum Methods $B$ (2008) 266:530-4.

15. Bassler N, Knudsen H, Moller SP, Petersen JB, Rahbek D, Uggerhoj UI. AD4/ACE collaboration: bubble detector measurements of a mixed radiation field from antiproton annihilation. Nucl Instrum Methods B (2006) 251:269-73. doi: 10.1016/j.nimb.2006.05.020

16. Sullivan AH. A measurement of the local energy deposition by antiprotons coming to rest in tissue-like material. Phys Med Biol. (1985) 30:1297-303. doi: 10.1088/0031-9155/30/12/003

17. Bassler N, Holzscheiter MH, Petersen J. Antiproton radiation dosimetry. Ion Beams Biol Med. (2007) 117-21.

18. Bassler N, Holzscheiter MH, Jäkel O, Knudsen HV, Kovacevic S. The antiproton depth-dose curve in water. Phys Med Biol. (2008) 53:793-805. doi: 10.1088/0031-9155/53/3/017

19. Bassler N, Hansen JW, Palmans H, Holzscheiter MH, Kovacevic S, AD4/ACE Collaboration: the antiproton depth-dose curve measured with alanine detectors. Nucl Instrum Methods B (2008) 266:929-36. doi: 10.1016/j.nimb.2008.01.067

20. Boll RM, Caccia M, Welsch CP, Holzscheiter MH. Using monolithic active pixel sensors for fast monitoring of therapeutic hadron beams. Radiat Meas. (2011) 46:1971-3. doi: 10.1016/j.radmeas.2011.05.053

21. Sellner S, Boll R, Caccia M, Negrini L, Straße T, Tegami S, et al. The antiproton cell experiment-do antiprotons offer advantages over other particle beam modalities? Hyperfine Interac. (2012) 213:159-74. doi: 10.1007/s10751-0120638-z

22. Bassler N, Holzscheiter M, Knudsen H. AD4/ACE Collaboration: cancer therapy with antiprotons. 8th international conference on low energy antiproton physics. Bonn. AIP Conf Proc. (2005) 796:423-30. doi: 10.1063/ 1.2130206

23. Raju MR, Bain E, Carpenter SG, Cox RA, Robertson JB. A heavy particle comparative study. Part II: cell survival versus depth. Br J Radiol. (1978) 51:704-11. doi: 10.1259/0007-1285-51-609-704

24. Raju MR, Amols HI, Bain E, Carpenter SG, Cox RA, Robertson JB. A heavy particle comparative study. Part III: OER and RBE. Br J Radiol. (1978) 51:712-9. doi: 10.1259/0007-1285-51-609-712

25. Withers HR, Lee SP, McBride W, Solberg TD, DeMarco JJ, Agazaryan N, et al. Potential for a radiotherapeutic gain from an anti-proton beam. 21st annual ESTRO meeting. Prague. Radiother Oncol. (2002) 64:S93. doi: 10.1016/S01678140(02)82595-6

26. Holzscheiter MH, Agazaryan N, Bassler N, Beyer G, DeMarco JJ, Doser M. Biological effectiveness of antiproton irradiation. Nucl Instrum Methods B (2004) 221:210-4. doi: 10.1016/j.nimb.2004.03.057

27. Maggiore C, Agazaryan N, Bassler N, Blackmore E, Beyer G, DeMarco JJ, et al. Biological effectiveness of antiproton annihilation. Nucl Instrum Methods B (2004) 214:181-5. doi: 10.1016/S0168-583X(03)01781-6

28. Bassler N, Holzscheiter M. Calculated LET spectrum from antiproton beams stopping in water. Acta Oncol. (2009) 48:223-6. doi: 10.1080/02841860802266730

29. Weber P. AD-4/ACE: Antiproton Cell Experiment. SSRMP Annual Scientific Meeting (Basel) Abstracts and Proceedings. (2009) 27.

30. Wouters BG, Lam GKY, Oelfke U, Gardey K, Durand RE, Skarsgard LD. Measurements of relative biological effectiveness of the $70 \mathrm{MeV}$ proton beam at TRIUMF using Chinese hamster V79 cells and the high-precision cell sorter assay. Radiat Res. (1996) 146:159-70. doi: 10.2307/3579588

31. Holzscheiter M, Alsner J, Angelopoulos A, Bassler N, Beyer G, Boll R, et al. Status Report for Experiment AD (2013). Annual report on AD-4 Experiments performed during 2012. CERN-SPSC-2013-001; SPSC-SR-111.

32. Fahimian B, DeMarco J, Holzscheiter M, Keyes R, Bassler N, Iwamoto K, et al. Antiproton therapy: monte carlo simulations of normal tissue equivalent dose from annihilation neutrons. 50th annual AAPM meeting. Houston TX. Med Phys. (2008) 35:2874. doi: 10.1118/1.2962384

33. Fahimian BP, DeMarco J, Keyes R, Bassler N, Iwamoto KS, Zankl M, et al. Antiproton radiotherapy: peripheral dose from secondary neutrons. Hyperfine Interact. (2009) 194:313-8. doi: 10.1007/s10751-009-0086-6

34. Xu XG, Bednarz B, Paganetti H. A review of dosimetry studies on externalbeam radiation treatment with respect to second cancer induction. Phys Med Biol. (2008) 53:R193-241. doi: 10.1088/0031-9155/53/13/R01

35. Xu XG, Paganetti H. Better radiation weighting factors for neutrons generated from proton treatment are needed. Radiat Prot Dosimetry (2010) 138:291-4. doi: $10.1093 / \mathrm{rpd} / \mathrm{ncq} 028$

36. Keyes RW, Luan S, Holzscheiter M. SU-FF-T-416: antiproton therapy: a simplified method to characterize and compare dose from peripheral radiation fields. Med Phys. (2009) 36:2617. doi: 10.1118/1.3181898

37. Kalogeropoulos TE, Muratore R. Antiprotons for imaging and therapy. Nucl Instrum Methods B (1989) 40-1:1322-5. doi: 10.1016/0168-583X(89)90648-4

38. Kantemiris I, Angelopoulos A, Bassler N, Giokaris N, Holzscheiter MH, Karaiskos $\mathrm{P}$, et al. AD-4/ACE collaboration: real-time imaging for dose evaluation during antiproton irradiation. Phys Med Biol. (2010) 55:N123-31. doi: $10.1088 / 0031-9155 / 55 / 5 /$ N01

39. Kantemiris I, Karaiskos P, Georgiou E, Angelopoulos A, Giokaris N, Dubnicka S, et al. On the feasibility of real time imaging in radiotherapy using antiproton beams. JINST (2009) 4:P06002. doi: 10.1088/1748-0221/4/ 06/P06002

40. Sellner S, Welsch CP, Holzscheiter MH. Real-time imaging of antiprotons stopping in biological targets - Novel uses of solid state detectors. Radiat Meas. (2011) 46:1770-2. doi: 10.1016/j.radmeas.2011.05.052

41. Parodi K, Enghardt W, Haberer T. In-beam PET measurements of beta+ radioactivity induced by proton beams. Phys Med Biol. (2002) 47:21-36. doi: 10.1088/0031-9155/47/1/302

42. Pönisch F, Parodi K, Hasch BG, Enghardt W. The modelling of positron emitter production and PET imaging during carbon ion therapy. Phys Med Biol. (2004) 49:5217-32. doi: 10.1088/0031-9155/49/23/002

43. Polf JC, Peterson S, McCleskey M, Roeder BT, Spiridon A, Beddar S, et al. Measurement and calculation of characteristic prompt gamma ray spectra emitted during proton irradiation. Phys Med Biol. (2009) 54:N519-27. doi: 10.1088/0031-9155/54/22/N02 
44. Angelopoulos A. Investigation of antiproton use for therapy and imaging. 4th international summer school on nuclear physics methods and accelerators in biology and medicine. AIP Conf Proc. (2007) 958:78-82. doi: $10.1063 / 1.2825837$

45. Bassler N, Kantemiris I, Karaiskos P, Engelke J, Holzscheiter MH, Petersen JB. Comparison of optimized single and multifield irradiation plans of antiproton, proton and carbon ion beams. Radiother Oncol. (2010) 95:87-93. doi: 10.1016/j.radonc.2010.02.026

46. Handley SM, Ahmad S. An antiproton simulation study using MCNPX for radiation therapy. J Xray Sci Technol. (2011) 19:345-53. doi: 10.3233/XST2011-0298

47. Suit H, DeLaney T, Goldberg S, Paganetti H, Clasie B, Gerweck L, et al. Proton vs carbon ion beams in the definitive radiation treatment of cancer patients. Radiother Oncol. (2010) 95:3-22. doi: 10.1016/j.radonc.2010.01.015

48. Agazaryan N, DeMarco JJ, Lee SP, Smathers JB, Solberg TD, Withers HR, et al. Investigation on the use of anti-protons in clinical radiotherapy. 21st annual ESTRO meeting. Prague. Radiother Oncol. (2002) 64:S52. doi: 10.1016/S01678140(02)82477-X

49. Fahimian B, DeMarco J, Keyes R, Luan S, Zankl M, Holzscheiter M. Antiproton radiotherapy: development of physically and biologically optimized monte carlo treatment planning systems for intensity and energy modulated delivery. 51st annual aapm meeting. Anaheim CA. Med Phys. (2009) 36:2758. doi: $10.1118 / 1.3182464$

50. Shmatov ML. The possibility of using antiproton annihilation on uranium and thorium nuclei for killing cancer cells. Tech Phys Lett. (2008) 34:335-7. doi: $10.1134 /$ S1063785008040196
51. Kossov M. Simulation of antiproton-nuclear annihilation at rest. IEEE Trans Nucl Sci. (2005) 52:2832-5. doi: 10.1109/TNS.2005.862878

52. Jackson GP. Practical uses of antiprotons. Hyperfine Interact. (2003) 146:319-23. doi: 10.1023/B:HYPE.0000004216.58268.c4

53. Lewis RA, Smith GA, Howe SD. Antiproton portable traps and medical applications. Hyperfine Interact. (1997) 109:155-64. doi: 10.1023/A:1012653416870

54. United States Patent 8109865. Jackson GP, Hbar Technologies. (2012).

Conflict of Interest Statement: The authors declare that the research was conducted in the absence of any commercial or financial relationships that could be construed as a potential conflict of interest.

Received: 27 October 2013; accepted: 27 December 2013; published online: 16 January 2014.

Citation: Bittner MI, Grosu AL, Wiedenmann N and Wilkens JJ (2014) A systematic review of antiproton radiotherapy. Front. Physics 1:37. doi: 10.3389/fphy.2013.00037 This article was submitted to Biomedical Physics, a section of the journal Frontiers in Physics.

Copyright (C) 2014 Bittner, Grosu, Wiedenmann and Wilkens. This is an openaccess article distributed under the terms of the Creative Commons Attribution License (CC BY). The use, distribution or reproduction in other forums is permitted, provided the original author(s) or licensor are credited and that the original publication in this journal is cited, in accordance with accepted academic practice. No use, distribution or reproduction is permitted which does not comply with these terms. 